\title{
The Way of Laryngoscope Disinfection: A Survey of Practice in Various Hospitals and Clinics
}

\author{
Rubaiat $\mathrm{MM}^{1}$, Lateef $\mathrm{KA}^{2}$, Quader $\mathrm{A}^{3}$, Khurshid $\mathrm{HM}^{4}$
}

\begin{abstract}
Background and Aims: During general anaesthesia, laryngoscope is a common equipment for intubation used by anaesthesiologists. Laryngoscope has been identified as a potential source of cross infection. Though guidelines exist for appropriate disinfection practices, recent reviews suggested that current methods of disinfection areless effective and compliance is poor with the established protocols. We conducted a questionnaire-based survey to study the current disinfection practices being followed by a cross section of anaesthesiologists.
\end{abstract}

Methods: A simple questionnaire containing 14 questions was distributed amongst anaesthesiologists through online as well as direct contact. Data were analyzed with percentage analysis.

Results: Out of 150 anaesthesiologists, 120 submitted the completed questionnaires. Residents constituted $41 \%$ and $46 \%$ were consultants. Eighteen (12\%) used only tap water for cleaning and 132 (88\%) used a chemical agent after rinsing with water. Out of 132, 76 (51\%) used detergent/soap solution, 29 (19\%) would wash and then soak in disinfectant or germicidal agents (glutaraldehyde, povidone iodine and chlorhexidine) and 18 (12\%) would wipe the blade with an alcohol swab. With respect to disinfection of laryngoscope handles, $70 \%$ respondents said they used an alcohol swab, $18 \%$ did not use any method, $9 \%$ were not aware of the method being used, while $3 \%$ did not respond.

Conclusion: Our results indicate wide variation in methods of decontamination of laryngoscopes. Awareness regarding laryngoscope as a potential source of infection was high. We need to standardize and implement guidelines on a national level and make available resources which will help to improve patient safety.

CBMJ 2020 January: vol. 09 no. 01 P: 4-10

Key words: Disinfection, laryngoscopes, semicritical equipment, survey

\section{Introduction}

Day to day practice in anaesthesia, laryngoscope is a common equipment used by anaesthesiologists for general anaesthesia and endotracheal intubation. It is a one of the source of cross infection because of blood and bacterial contamination. ${ }^{1,2}$ It is shown that wide range of microorganisms have grown on laryngoscope blades. ${ }^{3,4}$ Laryngoscopes are included in semicritical items according to the Centers for Disease Control's (CDC's) Healthcare Infection Control Practices Advisory Committee (HICPAC), which minimally require high-level disinfection using chemical disinfectants. Glutaraldehyde, hydrogen peroxide, orthophthalaldehyde and peracetic acid with hydrogen peroxide are approved by the Food and Drug Administration., ${ }^{3,4}$ We carried out a survey amongst anaesthesiologists working at various hospitals and clinic in Dhaka and Chittagong metropolitan areasto know their current practice of laryngoscope cleaning and disinfection techniques.

1. Dr. (Lt Col) Molla Md Rubaiat, DA FCPS (Anaesthesiology), Department of Anaesthesiology and Intensive Care Unit, Naval Base Hospital (CMH BNS Patenga), Chittagong

2. Professor (Col) Kazi Ashkar Lateef, FCPS (Anaesthesiology), CMH Dhaka

3. Dr. (Lt Col) Abdul Quader, (MS Ortho), Naval Base Hospital (CMH BNS Patenga)

4. Dr. (Lt Col) Husain Md Khurshid, DA FCPS (Anaesthesiology), CMH Ramu

Address of correspondence:

Email:mrubaiat@gmail.com

Mobile: 01769050735 
Methods:

We designed a simple questionnaire regarding disinfection of laryngoscopes after consulting with the senior anaesthesiologist. It was offered to anaesthesiologists who are working various government and private hospital. Participation was voluntary. Participants were assured of maintaining anonymity and confidentiality. The questionnaire included 14 questions [Appendix 1]. Participants were asked to comment based on their standard everyday practice. The study cohort included anaesthesiologists working in teaching hospitals, public hospitals, private nursing homes and corporate hospitals. From the responses obtained, data were analyzed with percentage analysis.

\section{Results:}

Out of 150 those who responded online, 120 submitted the completed questionnaire. Out of these 120 respondents, $60(50 \%)$ were consultants, $54(45 \%)$ were trainee in anaesthesiology and $06(5 \%)$ were medical officer (trained in anaesthesiology) [Fig: -1].

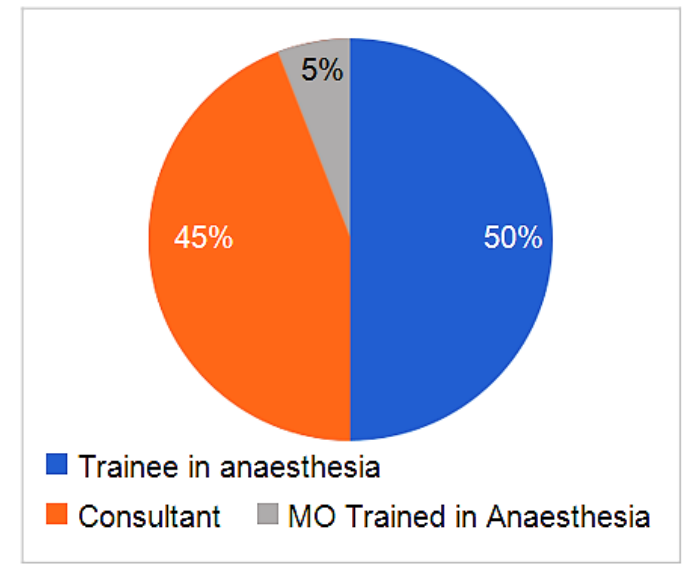

Fig-1: Designation of participating anaesthesiologist.

Sixty-eight (45\%) worked in teaching and public hospitals, 49 (33\%) practiced in Private hospital/nursing homes and 33 $(22 \%)$ in corporate hospitals [Fig: -2$]$.

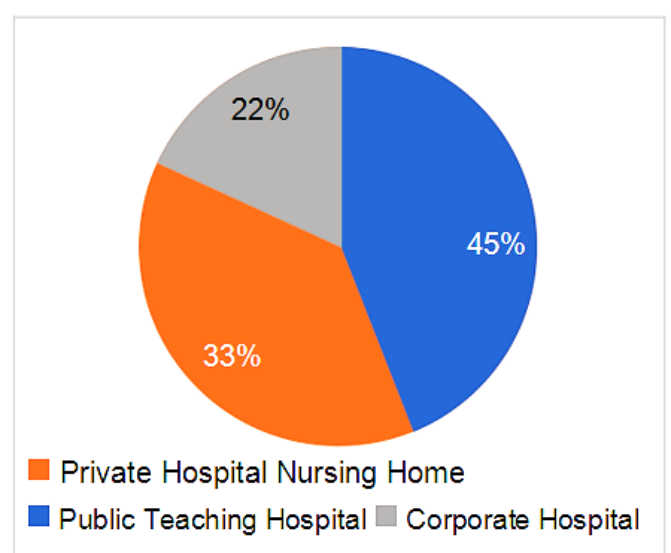

Fig-2: Working place of anaesthesiologist.

The study respondents performed 3 laryngoscopies/day (range1-5). On enquiring about who disinfected the laryngoscopes routinely, $66(55 \%)$ respondents said it was done by operation theatre (OT) technicians, $48(40 \%)$ said it was done by ward boys and $06(5 \%)$ said they cleaned the laryngoscopes themselves.

On asking about the method used for cleaning and disinfection of laryngoscope blades, $12(10 \%)$ respondents said they used only tap water for cleaning. One hundred eight (108) (90\%) used a chemical agent after rinsing with water. Out of 108,81 (75\%) used tap water initially followed by wipe with chlorhexidine + alcohol (eg. Hexisol), 15 (14\%) used detergent/soap solution followed by wiped with chlorhexidine, 09 (8\%) would wipe the blade with an alcohol swab and 03 (3\%) kept laryscopic blade immersed under glutaraldehyde (eg. Cidex) solution for 30 minutes. None of the respondents used autoclaving as a method for blade [fig; -3].

We did not find any difference regarding awareness of disinfection methods between residents and consultants. 


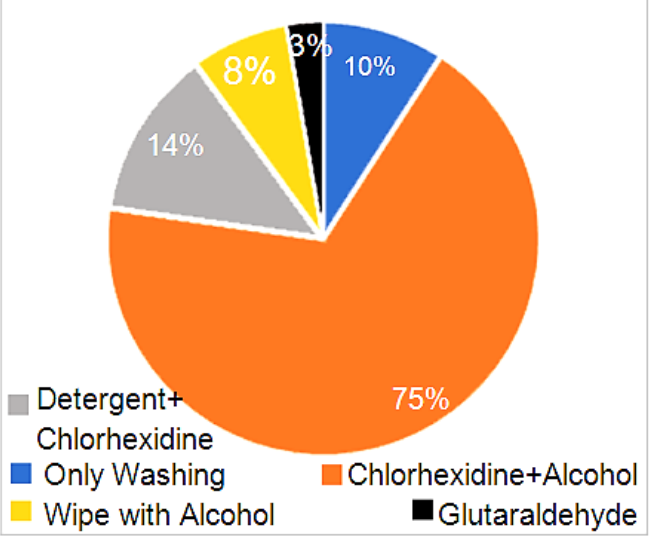

Fig-3: Methods of disinfection of laryngoscope blade

When asked regarding the frequency of disinfection of blades, $106 \quad$ (98\%) respondents said they did it after every case. Two (2\%) said some time they forgot to clean but finally clean it at the end of the day.

We also asked about frequency of cleaning and disinfection of the laryngoscope handle. Nearly $76 \%$ said they would clean it end of all operations, $20 \%$ would clean it after every operation and $4 \%$ would clean it occasionally [fig: -4].

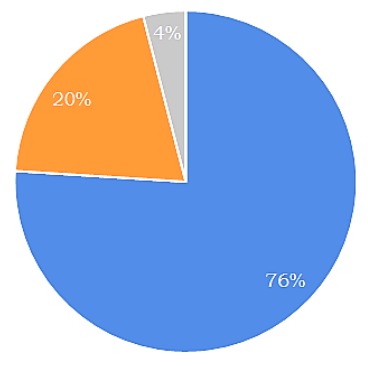

End of all operations $\quad$ After every operation Occationally

Fig-4: Disinfection of laryngeal handle.

On asking about the method of disinfection used for laryngoscope handles, nearly $70 \%$ respondents said they used chlorhexidine + alcohol (eg, Hexisol), 18\% used alcohol and
$9 \%$ used only chlorhexidine (eg, Savlon), while $3 \%$ did not respond.

Regarding how sterility of laryngoscopes was assured, $17 \%$ respondents said they used swab and culture method and $76 \%$ did not use any method. Almost $7 \%$ did not attempt this question.

On enquiring whether they had administered anaesthesia to infected patients, $97 \%$ of respondents said they had anaesthetized infected cases with Hepatitis B, respiratory infections (only emergency operation) and sometimes skin disease as a last case of operation.

On enquiring about the methods of decontamination of such infected laryngoscopes, nearly $24 \%$ used alcohol swab and $13 \%$ did not attempt the question. Nearly 35\% used chlorhexidine, 23\% used glutaraldehyde (eg cidex), 5\% used disposable laryngoscope, [fig: -5].

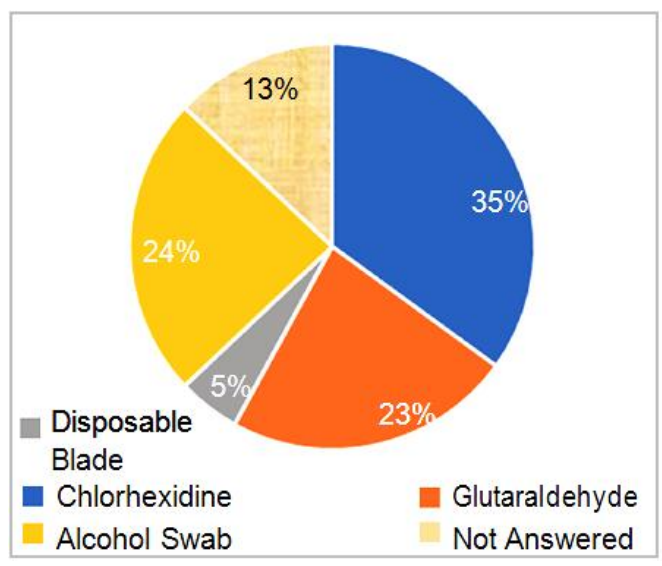

Fig-5: Decontamination of infected laryngoscope blade.

Five of the respondents commented that they liked the questionnaire and requested that the survey results are mailed to them.

\section{Discussion}

In general anaesthesia laryngoscopy is an invasive procedure involving contact of the laryngoscope with the mucous membrane, saliva, and at times even blood, making it a source of hospital-acquired infections. ${ }^{5,6}$

In 1986, CDC published guidelines for 
disinfection of equipment. They proposed that equipment which are in direct or indirect contact with mucus membrane or respiratory tract mucosa are classified as semi-critical and need a high level of disinfection which destroys all forms of microorganisms except bacterial spores. ${ }^{2,3}$ They suggested hot water pasteurization $\left(80^{\circ} \mathrm{C}-100^{\circ} \mathrm{C}\right.$, for 30 min) or exposure to Environmental Protection Agency-registered sterilant chemical for short exposure time (10-45 $\min )^{3,4,5}$ These methods have been universally accepted and supported by various organizations. ${ }^{3,4}$

The guidelines for preventing healthcareassociated pneumonia issued by CDC and HICPAC recommend a detailed procedure for cleaning and disinfecting laryngoscopes. They recommend thorough washing followed by disinfection with steam sterilization or wet heat pasteurization of these semicritical equipment. ${ }^{7}$ The recommended chemical disinfectants are high-level disinfectants such as ethanol (>70\%), formaldehyde, hypochlorite, orthophthalaldehyde and glutaraldehyde. ${ }^{4,7}$

Some review articles suggest ineffectiveness of current methods of disinfection of reusable laryngoscopes and poor compliance with the established protocols. $^{8,9}$

In Bangladesh, there is no uniformity of laryngoscope disinfection practices. In our hospital too, different OTs follow different practices for disinfection of laryngoscopes. In Bangladesh no such survey found on line. In 2010, a survey was carried out regarding existing practices of disinfection of laryngoscope in the Indian context. ${ }^{10}$ The results of the survey showed that there was no protocol for disinfection. The authors also compared bacterial growth on front, back and light bulb of laryngoscope blade washing with water and washing with water followed by disinfection with $5 \% \mathrm{v} / \mathrm{v}$ aldehyde-free biguanide agent. Fifty-eight per cent samples of washing with only water showed growth of pathogenic microorganisms, whereas $3.4 \%$ samples of biguanide group showed growth of commensals. The authors recommend disinfection with $5 \% \quad \mathrm{w} / \mathrm{v}$ aldehyde-free biguanide agent for at least $10 \mathrm{~min}$ as an effective and inexpensive method. In this study, a subset of patients with oral cancer showed significant bacterial growth (52\%) due to suboptimal hygiene. ${ }^{10}$ Their hospital changed the practice of sterilization of laryngoscopes as per the study result. ${ }^{10} \mathrm{~A}$ recent online survey of 100 anaesthesiologists from tertiary hospitals in India found that many respondents considered laryngoscopes as a potential source of infection. However, there was marked variability in decontamination methods. ${ }^{11}$ Our study results also corroborate the results of other studies. Awareness regarding the need for cleaning and disinfection of laryngoscopes was high (nearly $94 \%$ for blade; $89 \%$ for handle). However, practices differed greatly. ${ }^{11}$

Beside laryngoscopic blade, handle can also be a source of hospital infection. Though it is not come in direct contact with mucus membrane, but at closed position, the contaminated tip is always in contact with the handle. Various strategies to minimize contamination include improving methods of decontamination, use of disposable laryngoscopes or redesigning the handle to minimize contact points. ${ }^{12,13}$

Even after appropriate disinfection, improper handling methods can lead to recontamination of reusable laryngoscopes. Hence, along with standardization of decontamination methods, there is a need to review protocols and policies surrounding the use of reusable blades. ${ }^{14}$

Our study has some limitations. Our survey was limited to online. This survey was conducted in metro city and district level where adequate resources in the form of staff, disinfectant materials, equipment and well-equipped microbiology laboratories are most often readily available. Thana level government hospital, rural hospitals, nursing homes/clinics may lack these facilities. Standardization of protocols for disinfection and availability of resources for all will help in minimizing the risk of cross infection associated with laryngoscope blade and handles. 


\section{Conclusion}

Our survey showed a high level of awareness regarding methods of decontamination of laryngoscopes amongst anaesthesiologist. In Bangladesh, practices for cleaning and disinfection of laryngoscopes vary widely. National guidelines for decontamination of anaesthesia equipment are necessary. We need to standardize and implement guidelines on a national level and make available resources which will help to improve patient safety.

\section{APPENDIX 1 \\ Questionnaire}

Please tick your option.

1) Your designation:
a) Resident/trainee
b) Medical officer trained in anaesthesia
c) Consultant

2) I am practicing currently in:
a) Government hospital
b) Teaching institute
c) Corporate hospital/ Private hospital/clinic

3) Approximate number of patients in whom I use the same conventional laryngoscope blade per day:

4) In my practice, disinfection of anaesthesia equipment is done by:
a) OT technician
b) Ward boy
C) Any other

5) I use the following method for cleaning and disinfection of laryngoscope blade:
a) Rinse under running water
b) Washing with detergent/soap solution
c) Wipe with an alcohol swab

d) Soaking in disinfectant/germicidal agent

e) Autoclaving

f) Don't know

g) Others (specify)

6) If (d), how do you prepare the solution?
a) Don't know
a) Preformedsolutionname
b) Dilutionpreparedas

7) If (d), how much time is it kept in the solution?

8) I disinfect the blade:
a) After each patient
a) Only after an infected or high-risk patient
b) At the end of the day
c) Other

9) I disinfect the laryngoscope handle in the following way:
a) Wiping with alcohol swab
b) Any other method
C) Don't use any method

10) I disinfect the laryngoscope handle:
a) After every use
b) Occasionally
c) I don't disinfect the handle generally
d) At the end of all operations

11) How do you assure the sterility of the laryngoscope?
a) With the help of swab and culture
b) I don't use any method 
12. Have you ever administered anaesthesia to a patient infected with?
a) A respiratory infection
i) Yes
ii) No
b) Seropositive patient
i) Yes
ii) $\mathrm{No}$
c) Psoriasis or dermatitis
i) Yes
ii) No
d) Herpes simplex
i) Yes
ii) No
e) Other infection

13) If yes, do you take any extra precaution?

a) Disposable laryngoscope blade

b) Alternate method of sterilization (specify)

14) Any comments that you have practiced:

\section{References:}

1. Hall JR. Blood contamination of anesthesia equipment and monitoring equipment. AnesthAnalg. 1994;78:1136-9.

2 Morell RC, Ririe $D$, James $R L$, Crews $D A$, Huffstetler K. A survey of laryngoscope contamination at a university and a community hospital. Anesthesiology. 1994;80:960.

3 Bucx MJ, Dankert J, Beenhakker MM, Harrison TE. Decontamination of laryngoscopes in The Netherlands. $\mathrm{Br} \mathrm{J}$ Anaesth. 2001;86:99-102.

4 Centers for Disease Control. Guideline for handwashing and hospital environmental control, 1985.Section 2: Cleaning, disinfecting, and sterilizing patient-care equipment. Infect Control. 1986;7:236-40.

5 Beamer JE, Cox RA. MRSA contamination of a laryngoscope blade: A potential vector for cross infection. Anaesthesia. 1999;54:10101.

6 Orhan ME, Saygun O, Güzeldemir ME. An alternative simple method in laryngoscope blade decontamination. Middle East $J$ Anaesthesiol. 2002;16:529-34.

7 William A. Rutala, Ph.D., M.P.H. CDC Guideline for Disinfection and Sterilization in Healthcare Facilities, 2008 Update: May 2019;11-12, 106-109.

8 Machan MD. Infection control practices of laryngoscope blades: $A$ review of the literature. AANA J. 2012;80:274-8.

9 Negri de Sousa AC, Levy CE, Freitas MI. Laryngoscope blades and handles as sources of cross-infection: An integrative review. J Hosp Infect. 2013;83:269-75.

10 Telang R, Patil V, Ranganathan P, Kelkar R. Decontamination of laryngoscope blades: Is our practice adequate? J Postgrad Med. 2010;56:257-61.

11 Chawla R, Gupta A, Gupta A, Kumar M. Laryngoscope decontamination techniques: $A$ survey. I Anaesthesiol Clin Pharmacol. 2016;32:99-102. 
Original Article

12. Call TR, Auerbach FJ, Riddell SW, Kiska DL, Thongrod SC, Tham SW, et al. Nosocomial contamination of laryngoscope handles: Challenging current guidelines. Anesth Analg. 2009;109:479-83.

13. Simmons SA. Laryngoscope handles: $A$ potential for infection. AANA J. 2000;68:2336.

14. Lowman W, Venter L, Scribante J. Bacterial contamination of re-usable laryngoscope blades during the course of daily anaesthetic practice. S Afr Med J. 2013;103:386-9. 